\title{
Semdrops: A Social Semantic Tagging Approach for Emerging Semantic Data
}

\author{
Diego Torres, Alicia Diaz \\ LIFIA - Facultad de Informática - UNLP \\ La Plata, Argentina \\ \{diego.torres, alicia.diaz\}@lifia.info.unlp.edu.ar
}

\author{
Hala Skaf-Molli, Pascal Molli \\ Lina - University of Nantes \\ Nantes, France \\ \{Hala.Skaf, Pascal.Molli\}@univ-nantes.fr
}

\begin{abstract}
This paper proposes a collective intelligence strategy for emerging semantic data. It presents a combination of social web practices with semantic web technologies to enrich existing web resources with semantic data. The paper introduces a social semantic tagging approach called Semdrops. Semdrops defines a conceptual model which is an extension of the Gruber's tag model where the tag concept is extended to semantic tag. Semdrops is implemented as a Firefox add-on tool that turns the web browser into a collaborative semantic data editor. To validate Semdrops's approach, we conducted an evaluation and usability studies and compared the results with automatic generation methods of semantic data such as DBpedia. The studies demonstrated that Semdrops is an effective and complementary approach to produce adequate semantic data on the Web.
\end{abstract}

Keywords-Social Tagging; Semantic Data; Semantic Social Tagging

\section{INTRODUCTION}

"The Semantic Web is an extension of the current web in which information is given well-defined meaning, better enabling computers and people to work in cooperation" [1]. Web resources of semantic web are semantically annotated with special meta-data that represent formally the meaning. The availability of this machine-readable meta-data enable intelligent access of Web resources by automated agents, adequate queries and combination of several sources of information [1].

Ontologies form the backbone of the Semantic Web systems and they are the means to semantically annotate Web resources. Due to the wide variety of topics presented on the web, existing ontologies partially cover a small part of the current web. For instance, in the fields of medicine, computers, society and business, a lot of efforts have been done to develop ontologies [2], [3], [4], but then quickly decreases in other domains. These ontologies are developed by domain experts resulting in sophisticated ontologies that are used in specific applications beyond the Web. Besides, developing and combing large ontologies involve sophisticated tasks.

Recently, there is a shift towards the development of lightweight ontologies for specific domains because the definition and combination of them are easier than large and sophisticated ones. Consequently, these lightweight ontologies are widely used in Semantic Web applications. Some popular examples are FOAF ${ }^{1}, \mathrm{SKOS}^{2}$ and $\mathrm{SIOC}^{3}$.

Although existing lightweight ontologies are defined by domain experts, many studies have demonstrated the significance of taking advantage of the social force to contribute to the semantic web vision [5], [6]. In this sense, it is important to provide users with tools that support the emergence of semantic data. Semantic data are the seed to make emerge lightweight ontologies. Semantic wikis [7], [8], [9] enable the emergence of lightweight domain ontologies by the community efforts. However, the emerged ontologies are limited to the Semantic Wiki scope and cannot be transparently extended to any Web resource.

Our objective is to provide a tool that allow the emergence of semantic data without being limited to semantic wikis. We propose an approach that allow the emergency of semantic data by means of identification and modelling of the tagging activity. This is a step to the semantification of the web based on social effort. The proposed approach has:

- to promote semantic data proliferation.

- to promote the semantic data quality

- to be applicable to any web resources.

- to be used by a wide community

- to provide an easy interaction mode.

In order to achieve all of these requirements, the challenge now is to define a simple semantic social tagging model whose integration into the system provides an easy interaction modality that enables to attach semantic tags to a web resource without modifying the resource itself. In this work, we propose a social semantic tagging approach called Semdrops. Semdrops defines a conceptual model which is an extension of the Gruber tag model [10], where the tag concept is extended to semantic tag. We implement Semdrops as a Firefox add-on tool that turns the web browser into a collaborative semantic data editor in situ for any web resource.

To validate Semdrops's approaches, we conducted an evaluation and usability studies and we compared the results

\footnotetext{
${ }^{1}$ http://www.foaf-project.org/

${ }^{2}$ http://www.w3.org/2004/02/skos/

${ }^{3}$ http://sioc-project.org/
} 
with automatic generation methods of semantic data such as DBpedia. The studies demonstrated that Semdrops is an effective and complementary approach to produce adequate semantic data on the Web.

This paper is organized as follow. Section II details the social semantic approach and defines the conceptual model of Semdrops. Section III details Semdrops the architecture and implementation. Section IV presents an evaluation and usability studies. Section V discusses the results of the evaluation. Section VI presents related works. Finally, section VII presents conclusions and future work.

\section{SEMDROPS APPROACH}

Semdrops uses the force of mass collaboration for facilitating the emergence of semantic data. This data can be used to emerge lightweight ontology. It is based on social semantic tagging strategy [11] that adopts the characteristics of social tagging enhanced with semantic meta-data. In order to make easy the semantic annotation activity to the user, Semdrops proposes a simple semantic representational model to conceptualise the social semantic tagging activity, as it is shown in section II-A. This model captures the semantic annotations performed by the users. This means that the model allows to represent the attachment of social semantic tags to a web resource in an easy manner. The model also enables tagging a resource without modifying the resource itself. Section II-C presents a detailed example.

Although, Semdrops social semantic tag model was designed to make easy the tagging activity by regular users, it has to be mapped to existing semantic representation models like RDF, OWL in order to make it compatible with the semantic web technologies. In this work, we particularly use Semantic MediaWiki to make this mapping as is detailed in the section II-B.

\section{A. Semantic Social Tagging Model}

According to the literature, the most elementary building blocks of a tagging model is a tripartite model [12] makes up of the taggers, the tags and the resources being tagged. Gruber in [10] introduced to model one more element, the source of the tags, and named it the four-places relation model. Semdrops conceptual model is an extension of the four-places relation one, where the tag concept is extended to semantic tag. Semdrops defines four main concepts: Web resource, tagger, semantic tag and semantic support.

Web resource. A Web resource is any Resource in the web which could be identifiable by an URI.

Tagger. The tagger is the responsible of adding semantic tags to the web resource. A tagger can be an individual user or a community.

Semantic Tags. According to mc sharaefel et al.[13], a semantic tag is one where the tag itself is backed up by an RDF. Semantic tags are semantic annotations used to add a semantic description over a Web resource in an

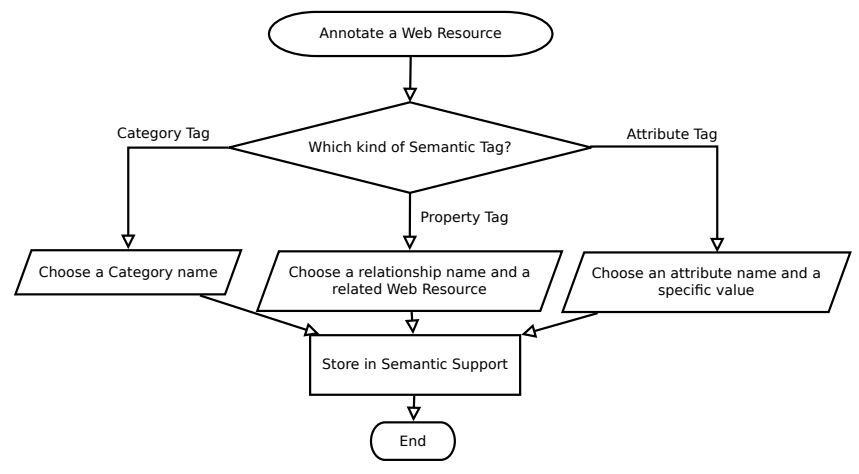

Figure 1. Tagging Flow

unsophisticated manner. . In our approach, we extend the definition of mc sharaefel et al.; particularly, we describe three kinds of semantic tags: Category Tag (CT), Property Tag (PT) and Attribute Tag (AT).

- Category Tag (CT).This semantic tag represents the belonging of a Web Resources to a Category. Tagging a resource by means of a CT defines the name of the tag as identifier of the category and the Web Resource as individuals which belongs to that category. Also, it is possible to organise categories specifying detailed levels by means of hierarchies using subsumption. A sub-category is defined as a subset of individuals of the super-category.

- Property Tag (PT). This semantic tag describes a relationship between two Web Resources. In this case, the name of the property types the relationship between the two web resources, even there are not a navigational link between them. Having PT, the navigation is improved by a semantic relationship.

- Attribute Tag (AT). This semantic tag describes a structural attribute for a web resource, for example the height of a mountain. It uses the label as name for the attribute and it is related with a simple data type as a number or a literal.

Semantic Support. We use this support to store semantic tags. The underlying semantic support has an impact on how Semdrops maps semantic tags to an existing semantic support data model. This is done by specifying a set of mapping rules. This semantic support makes Semdrops model compatible with other semantic web technologies. It can be any RDF repository e.g. Sesame [14], Jena [15] or even Semantic Mediawiki [7].

\section{B. Using Semantic Mediawiki as Semantic Support}

In this work, we particularly use Semantic MediaWiki (SMW) as a semantic support. We have chosen SMW because on one hand we have already used semantic wikis for knowledge personalisation [16] and on the other hand 


\begin{tabular}{|l|l|l|}
\hline Semdrops & SMW & OWL-like \\
\hline Category(X,TName $)$ & {$[[$ Category: TName $]$} & X rdf:type TName \\
\hline Property(X,PName,OW $)$ & {$[[$ PName::OWContent $]$} & X PName OW \\
\hline Attribute(X,AName,Value $)$ & $[$ [AName:= Value $]]$ & X AName Value \\
\hline
\end{tabular}

Table I

SEMDROPS MAPPING RULES

this allows using the powerful features of SMW e.g. query semantic tags and export semantic data.

Mapping Rules: We define a set of rules to transform Semdrops's semantic tags into suitable SMW semantic annotations in order to store them in the semantic support of SMW. In order to carry out this mapping, a web resource is mapped to a wiki article in terms of SMW.

- Category Semantic Tag. Let X be the URL identifying the Web Resource, and CatName the label for a category.

CategoryTag $(X$, CatName $) \equiv$ SemWiki $(X) \leftarrow([[$ Category : CatName $]])$.

Where SemWiki(page) $\leftarrow$ (code) means that the wiki page titled page has the string code included in its content.

- Property Semantic Tag. Let X, Y be the URLs identifying two different web resources and let PropName be the label of a property.

PropertyTag (X, PropName,OWebContent $) \equiv$ SemWiki $(X) \leftarrow([[$ PropName :: OWebContent $]])$

- Attribute Semantic Tag. Let X be the URL identifying a web resource and let AtrName be the label of an attribute and AValue can be a literal or numerical value. AttributeTag $(X$, AtrName, AValue $) \equiv$ $\operatorname{SemWiki}(X) \leftarrow([[$ AtrName $:=$ AValue $)]]$

The table I summarizes the mapping rules. The left hand is the Semdrops semantic tags, in the middle the equivalent SMW and the right the equivalent in owl-like.

\section{How to Use the Model?}

In this example, we consider a user who is reading the Paris city page ${ }^{4}$ on Wikipedia. She would like to tag that Paris is a City and also it is the location of the movie Amelie.

Following the workflow in figure 1, first she has to decide which kind of semantic tags want to use. She uses a Category Tag to describe Paris as a City. For that, the literal City is used as the name of the Category Tag. Next, she decides to use Property Tag to describe the relationship between Paris and the film Amelie. A Property Tag requires a label to type the relationship and also a URI for two involved resources: the movie and the city. Therefore the string was movie location of types the relationships and the Amelie Wikipedia's page represents the movie. Figure 2 (a) shows the initial wikipedia page and the figure 2 (b) shows the

\footnotetext{
${ }^{4}$ http://en.wikipedia.org/wiki/Paris
}

tagged page. The left side of the screen shows the semantic tags for the current page. The semantic tags tree has three main labels: Category, Links and Attributes. The user can visualize the semantic tags grouped by these three types and can fold and unfold the list of each group of tags.

During the semantic tagging activity, Semdrops maps the semantic tags into the semantic support model of SMW by using the mapping rules detailed in the previous section. All the new meta-data is generated without modifying the original resource.

In this scenario, a new semantic relation is created between Paris city and the Amelie movie, there was no prior relationship between these two resources even a navigational link.

1) Semdrops as Mashups Tool: The previous scenario shows how semantic tags can be added easily to a web resource. For instance, the semantic tags describing the web page of Paris in Wikipedia could be associated with those defined in the Paris wiki page at SemanticWeb.org ${ }^{5}$. In order to combine different sources, Semdrops includes the ability to access linked semantic data in cross semantic repositories. It takes special attention in owl:sameAs tags in the RDF definition of the web resource. For example, if in the RDF description of a web resource appears the tag owl:sameAs:: targetResource, the semantic tags of the targetResource will be added as semantic tags of the original resource.

Figure 2 (b) shows the use of Semdrops with the semantic tags of the example in the section II-C with a cross reference to the Paris page on SemanticWeb.org. In this example, the semantic tags with the green icon are from the cross reference, and the category City has a special icon because it was produced in both semantic repositories: SMW repository and the SemanticWeb.org repository.

\section{IMPLEMENTATION}

Semdrops uses a Model-View-Controller architecture as it is detailed in the figure 3. It includes a semantic MediaWiki as a semantic support for storing and retrieving the semantic tags.

Semdrops is implemented as a Firefox sidebar add-on, it is available as an open source and can be downloaded from http://sourceforge.net/projects/semdrops/. It implements the semantic social tagging model. The semantic tags tree has three main labels: Category, Links and Attributes. The user can visualize the semantic tags grouped by these three types and can fold and unfold the list of each group of tags as shown in the figure 2. Semdrops implements drag \& drop functionality, this improves user interaction and facilitates semantic tags generation. For instance, in the example of figure 2, a user can drag the France link and drop it on the Links label. This action opens the new link dialog box

\footnotetext{
${ }^{5}$ SemanticWeb.org is a Semantic Mediawiki used mainly for content related with Semantic Web
} 


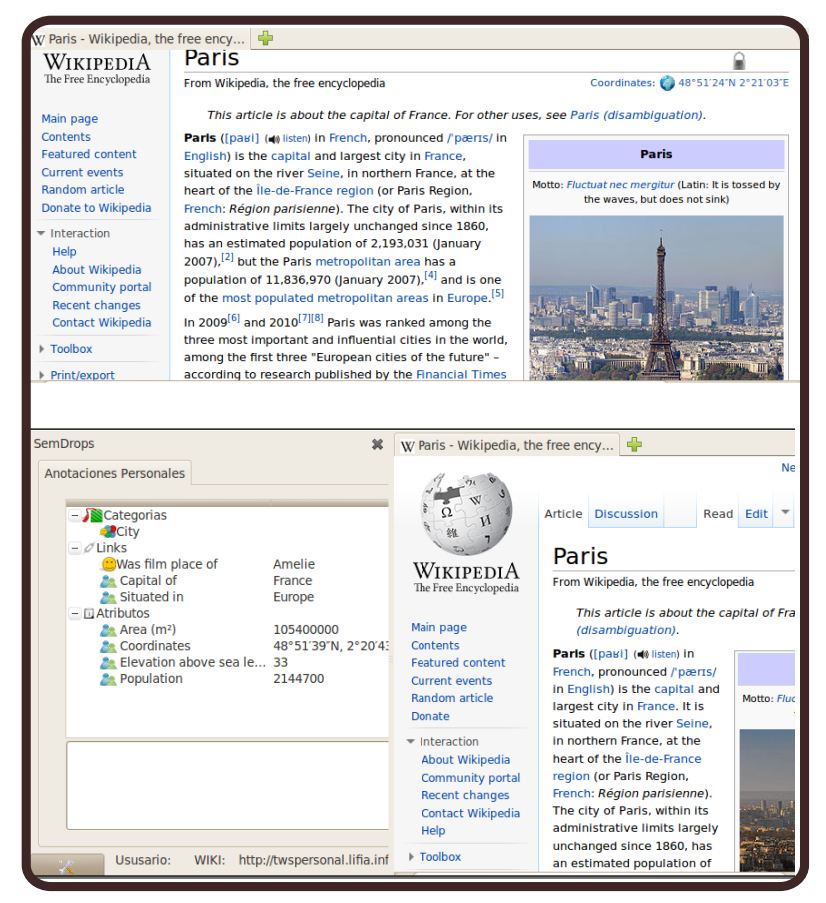

Figure 2. Semantic Tags for Wikipedia's Paris page: (a) On the top, the Wikipedia's Paris page without the Social Semantic Tags. (b) On the bottom, Semdrops in action for the same Wikipedia page.

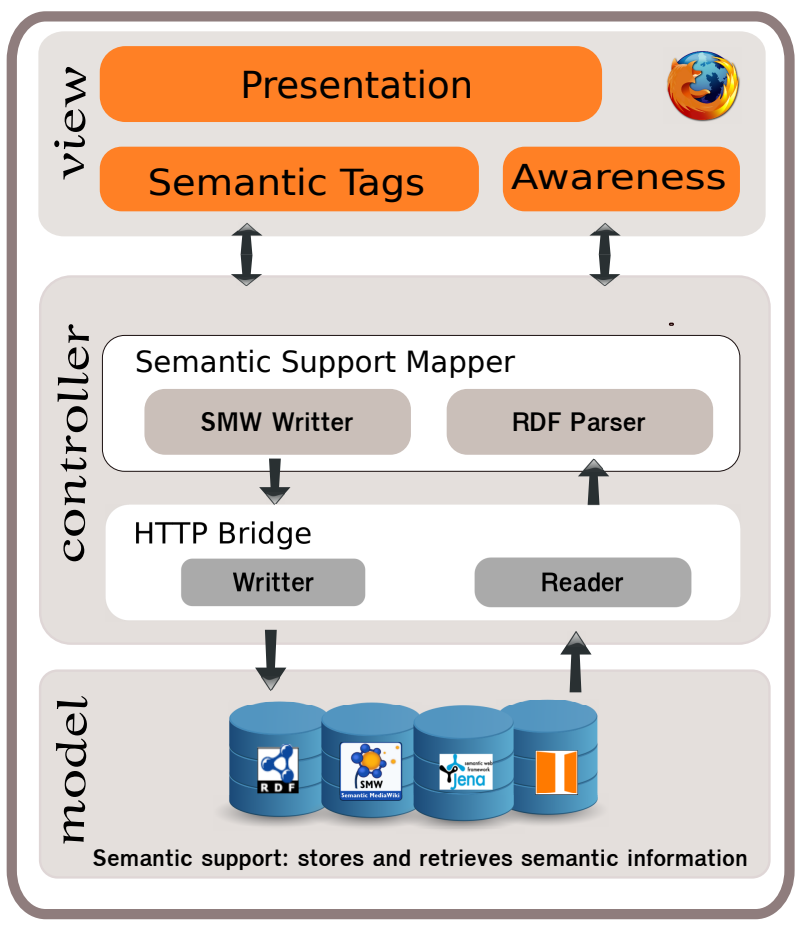

Figure 3. Semdrops Architecture filled with the France page link. Drag \& drop functionality is available for links and any text in the Web resource.

\section{Evaluation STUdy}

To demonstrate the capability of Semdrops to produce adequate semantic data, we conducted an evaluation study. In this study, we have asked a group of participants to use Semdrops to semantically tags a set of predefined web resources; more precisely, a set of Wikipedia pages. We have conducted also an usability study to measure the satisfaction of participants regarding Semdrops.

In the following sections, we detail the evaluation methodology, the qualitative and quantitative analysis and the usability study.

\section{A. Evaluation Methodology}

We have asked 20 people to participate to the evaluation, 15 males and 5 females. They are between 23 and 35 years old and fans of the TV Lost serie ${ }^{6}$, most of them are in the domain of computer sciences. In order to familiarize the participants with Semdrops, we have provided them a textual user guide and a screen-cast showing a using example ${ }^{7}$.

Every participant was able to use Semdrops to add semantic tags to two specific web resources: the Wikipedia pages Jhon Locke (Lost) ${ }^{8}$ and Richard Alpert (Lost) ${ }^{9}$, at any time and during four days. The participants were in different places and did not communicate with each other during the evaluation. Additionally, they only know that other people were involved in evaluation but they do not know who they were because we do not want the participants to communicate outside the system.

The participants were asked to read and analyse the wiki pages and after that add the semantic tags if they consider appropriate to semantically describe these wiki pages. They could also either delete or revise the semantic tags which they consider inappropriate. English language was used to name the tags in order to compare the results later with DBpedia.

\section{B. Evaluation Results}

1) Quantitative Analysis - Number of coincident tags with DBPedia: We manually compare Semdrops semantic tags with semantic information coming from the DBpedia database for the same web resources. The figure 4 represents a comparison between the set of the semantic tags added by Semdrops and that semantic information coming from the DBpedia database. Numbers in the figure represents the amount of semantic annotations. To make the manual comparison, we preprocessed the semantic information from

\footnotetext{
${ }^{6} \mathrm{http} / / /$ abc.go.com/shows/lost

${ }^{7}$ The screen cast is available at http://www.youtube.com/watch? $\mathrm{v}=$ N3Lr32iA9Vw

${ }^{8}$ http://en.wikipedia.org/wiki/John_Locke_(Lost)

${ }^{9}$ http://en.wikipedia.org/wiki/Richard_Alpert_(Lost)
} 


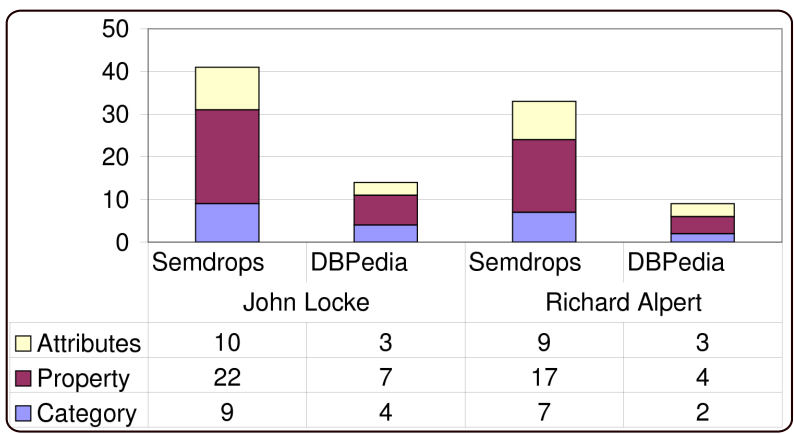

Figure 4. Quantities of semantic Tags

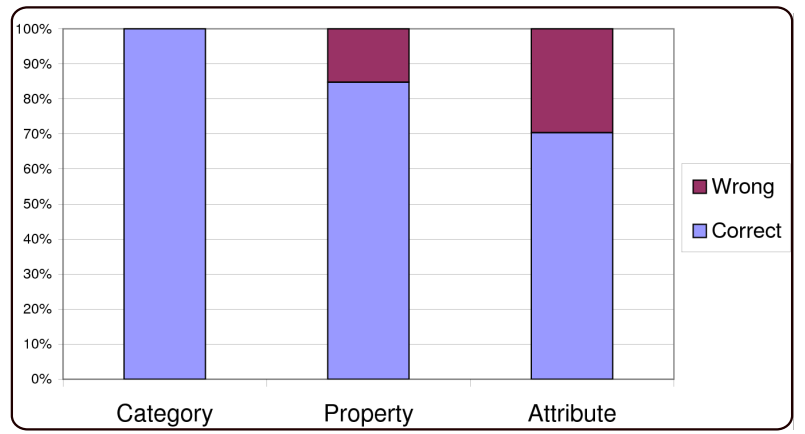

Figure 5. Quality of Semantic Tags

DBpedia to fix it to the Semdrops conceptual model, for example, skos:subject category: Fictional_Hunter in DBpedia match to a Semdrops Category Tag called Fictional Hunter.

For both considered Wikipedia pages (Jhon Locke and Richard Alpert ), we observed that:

- In general, the number of semantic tags added by the social effort using Semdrops was the double of the number of semantic tags that the automatically generated by DBpedia project.

- The most significant augmentation is the number of Property Tags. It was three times more in both Wikipedia pages.

2) Qualitative analysis: This analysis is in charge of finding out whether the participants have used the Semdrops conceptual model properly and made the right conceptualisation. This reveals the right understanding of the meaning of the category, property and attributes primitives by the participants. To do this, we manually inspected the Semdrops $\log$ file and observed that:

- All categories were well defined. This means that participants did not have any problem with the use of categories. They use the category tag according to the definition given in the section II.

- Sometimes the participants confuse the use of property and attribute tag. For example, some participants use birthday with value on May 30, 1956 as a Property tag instead of an Attribute tag.

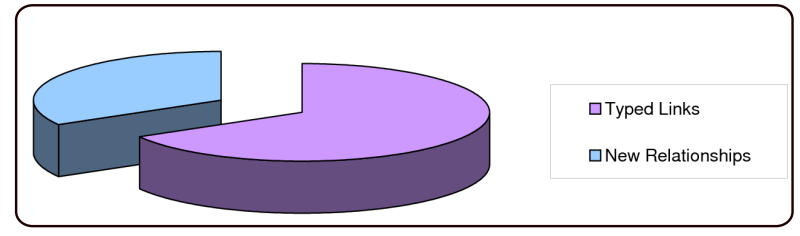

Figure 6. Proportion of Semantic Tags

- There were other cases where the right conceptualization might be controversial due to the information given in the wiki page was not enough (either because the abstraction level was not evident or the related concept was difficult to be expressed in a Wiki). Consequently, some attributes were not clear to be either an attribute or a property tag, we considered these cases also as not well defined.

To summarize, the rate of wrong property tags is near to $15 \%$, while it increases to $30 \%$ in the case of attributes as shown in the figure 5 .

3) Added Value Analysis: By analysing the kind of contribution of participants, we observed that:

- Most participants were worry about adding meaning to the navigational links, $67 \%$ of the property tags were defined over existing navigational links. For example, the navigational link between the John Locke and the Anthony Cooper Wikipedias pages was typed with son of property, representing that John Locke is the son of Anthony Cooper.

- In addition, new relationships among other resources were added. The $33 \%$ of the added property tags establish new links with other Wikipedia pages. This result is illustrated in the figure 6 .

It is important to notice that the enrichment of the existing content, in terms of both navigability and meaning, was possible to achieve by human interactions.

- At the first glance, the number of Categories defined with Semdrops seems to be higher of those defined in the initial Wikipedia pages. However, we later discovered that the most Semdrops categories already exist in Wikipedia. This was due to in Wikipedia the categories are organized in a hierarchy structure and only the most specific ones are shown in the wiki pages.

- Nearly $90 \%$ of Categories defined with Semdrops were related each other and could be organized in a hierarchy. Although, the subsumption property is part of the proposed semantic tag model, the current early version of Semdrops prototype does not support it. However, this result encourage us to extend Semdrops implementation to incorporate it.

To summarize, DBPedia has a good description of Category hierarchies and structural information of the page from Wikipedias infoboxes. On the other hand, Semdrops 
is better to make emerge semantic data that is not easy to obtain automatically. Both kind of semantic data are highly complementary.

\section{Usability Evaluation}

At the end of the evaluation, we made a set of questions to measure the satisfaction of the participants of the system. We use the System Usability Scale (SUS) of Brooke [17]. SUS is a Likert scale simple questionnaire giving a subjective global value of usability. The obtained values indicate that the average of SUS usability was 71.5 over 100 with a standard deviation of 8.6. This results show that the participants had acceptable satisfaction of the system [18].

In addition, we asked users the level of comfort for making category, property and attribute tags. Most of users had felt comfortable with the creation of categories and properties but less comfortable with the use of attributes.

\section{DISCUSSION AND LEARNED LESSONS}

The conducted evaluation shows that semantic data obtained by using Semdrops are complementary with those of DBpedia. DBpedia has semantic data related to the structural content of the wikipages i.e. most properties reached in Wikipedia are related with navigational structure, for example is dbprop:redirect of or is dbprop:disambiguates of or infoboxes. While, Semdrops promotes semantic data proliferation related to the nature of the wiki article based on users background.

In addition, during the evaluation, we notice that users created properties following three main patterns :

- semantic enrichment of existing navigational links by adding semantic types.

- new properties were defined by using information in wikipage that were not related to a navigational link.

- emergence of new properties that relate pages that do not have any reference in the content of the wikipage.

Other interesting point was the generation of attributes. Attributes tags had the highest rate of mistakes. In most cases this happened because for the tagger was not clear the difference between an attribute and a property tag.

Finally, by the manual examination of the log file, we notice that some semantic tags were rectified by users. For example, the spelling of the word bald to describe Jhon Locke character was rectified twice by different users each time. There is a community regulation similar to that present in wiki community practices. In this evaluation, we made manual analysis of the log file, an automatic one could help to consolidate the generated information.

\section{RELATED WORK}

In the last years, many efforts focus on the semantification of the web. One of the most relevant is the Linking Open Data (LOD) ${ }^{10}$ initiative. The objective of LOD is to connect

\footnotetext{
${ }^{10}$ http://linkeddata.org
}

related data that was not previously connected using URI and RDF. LOD generates a large amount of RDF statements, most of them are generated automatically, only less than $1 \%$ of semantic contents are a user-generated [19]. DBPedia [20] is a major application of LOD. DBpedia is the Linked Data version of Wikipedia, it is based on an automatic approach to generate RDF statements, by converting structured information in Wikipedia pages into RDF statements. In Semdrops approach, the semantic data emerge by the social effort.

Other approaches to increase the semantic data on the web are based on the enrichment of social tags with semantic information:

- Annotea [21] defines a simple ontology to generate semantic bookmarks.

- In [22], semantic bookmarks are generated automatically to structure hypermedia documents. This approach is limited to specific web resources i.e. the bookmarks. In Semdrops, semantic tags are not restricted to semantic bookmarks; they can be added to any web resource.

- SOBOLEO ${ }^{11}$ [23] uses SKOS taxonomy to annotate bookmarks. Although, SOBOLEO allows assigning tags to web resources and organize them into hierarchies, it does not support a rich semantic model.

- In Limpenss Ph.D. thesis[24] and Monin et al. [25] is introduced the niceTag ontology which subclassifies the isRelatedTo tag into a set of sub-properties to conceptualize the different possible uses of tags presented by Golder \& Huberman. Instead, Semdrops defines an open structure through which new properties definition can be added by the users.

- Huynh-Kim-Ban et al. [26] introduced an extension of the tag model presented in del.icio.us which includes both synonymy and inclusion relationships among tags, but they do not include the possibility to express, for example, attributes as Semdrops does.

OntoGame [27] combines Web 2.0 and semantic definition to determine correctly class-instance correspondence and super class or instance-of relations for Wikipedia articles. This work was very useful to define the building block of Semdrops semantic social tagging model, however, the last one defines a more rich semantic model.

Some other approaches propose semantical enrichment of social tagging [28], [10] by defining a tag ontology for this purpose. For instance, TagOntology [12] proposed a tripartite system (User, Resource, Tag); MOAT [29] allows the use of a URI to define the meaning of a tag, and SCOT [11] enforces the social context involved in folksonomies. All of these approaches focus on giving semantic to the tags. These model can be reused to give semantic to the Semdrops tags.

Other approach proposes to use semantic annotations to enrich wikis systems; resulting in semantic wiki systems. Semantic wikis [7], [8], [9] enable the emergence

\footnotetext{
${ }^{11}$ Social Bookmarking and Lightweight Engineering of Ontologies
} 
of lightweight domain ontologies by the community efforts. However, the emerged ontologies are limited to the Semantic Wiki scope and cannot be transparently extended to any Web resource. Semdrops is inspired in the semantic wikis approach but goes beyond allowing the semantic annotation of existing web content transparently.

\section{CONCLUSIONS AND FURTHER WORK}

This paper introduced a social semantic tagging approach called Semdrops. Semdrops defines a simple and rich social semantic tagging model. This model is implemented as a Firefox add-on tool that turns the web browser into a collaborative semantic data editor in situ for any web resource. The evaluation and usability studies demonstrated that Semdrops :

- promotes semantic data proliferation thanks to its simple social semantic tagging model.

- promotes the semantic data quality by community regulation.

- is applicable to any web resources thanks for using URI to identify them.

- can be used by a wide community thanks to its simple social semantic tagging and its implementation as a Firefox add-on.

- provides an easy interaction mode as demonstrated the usability study.

As future works, in order to consolidate results we will conduct a bigger evaluation with more resources and we will compare results with other work such as [30]. Also, we will evaluate how lightweight ontologies can emerge from the semantic data. For this propose we will profit from the existence of the subsumption concept in our model. Further, we will study inconsistency and conflict problems.

For a more pragmatical point of view, Semdrops needs to be extended to make the captured semantic data more usable in the context of the semantic web. For instance, Semdrops should adopt the LOD philosophy to share semantic data, and even it could incorporates properties already existing in the semantic web technologies like the sameAs property.

Besides, we are interesting to apply Semdrops in a more general scenario where social tagging is combined with personal one. In this sense, it is needed to extend Semdrops approach to support personal tagging.

\section{ACKNOWLEDGMENT}

This work was partially funded by: the Laccir R1210LAC007 project, and by the PAE 37279-PICT 02203 which is sponsored by the ANPCyT, Argentina.

\section{REFERENCES}

[1] T. Berners-Lee, J. Hendler, and O. Lassila, "The semantic web," Scientific American, vol. 284, no. 5, pp. 34-43, 2001.
[2] M. Hepp, "Goodrelations: An ontology for describing products and services offers on the web," in Proceedings of the 16th International Conference on Knowledge Engineering and Knowledge Management (EKAW2008), ser. Lecture Notes in Computer Science, A. Gangemi and J. Euzenat, Eds., vol. 5268. Springer, September 29 - October 3 2008, pp. 329 346.

[3] J. G. Breslin, A. Harth, U. Bojars, and S. Decker, "Towards semantically-interlinked online communities," in The 2nd European Semantic Web Conference (ESWC 05), ser. Lecture Notes in Computer Science, A. Gómez-Pérez and J. Euzenat, Eds., vol. 3532. Springer, May 29 - June 1 2005, pp. 500514.

[4] A. Miles and J. R. Pérez-Agüera, "Skos: Simple knowledge organisation for the web skos: Simple knowledge organisation for the web," Cataloging Classification Quarterly, vol. 43, no. 3, pp. 69-83, 2007.

[5] K. Siorpaes and E. P. B. Simperl, "Human intelligence in the process of semantic content creation," World Wide Web, vol. 13, no. 1-2, pp. 33-59, 2010.

[6] G. Hope, T. G. Wang, and S. Barkataki, "Convergence of web 2.0 and semantic web: A semantic tagging and searching system for creating and searching blogs," in Proceedings of the International Conference on Semantic Computing. Washington, DC, USA: IEEE Computer Society, 2007, pp. 201-208.

[7] M. Krötzsch, D. Vrandecic, M. Völkel, H. Haller, and R. Studer, "Semantic wikipedia," Journal of Web Semantic, vol. 5, no. 4, pp. 251-261, 2007.

[8] S. Auer, S. Dietzold, and T. Riechert, "Ontowiki - a tool for social, semantic collaboration," in International Semantic Web Conference, ser. LNCS, I. F. Cruz, S. Decker, D. Allemang, C. Preist, D. Schwabe, P. Mika, M. Uschold, and L. Aroyo, Eds., vol. 4273, 2006, pp. 736-749.

[9] M. Buffa, F. L. Gandon, G. Ereteo, P. Sander, and C. Faron, "Sweetwiki: A semantic wiki," Journal of Web Semantics, vol. 6, no. 1, pp. 84-97, 2008.

[10] T. Gruber, "Ontology of folksonomy: A mash-up of apples and oranges," Intl Journal on Semantic Web Information Systems, vol. 3, no. 2, pp. 1-7, 2007.

[11] H.-L. Kim, J. Breslin, S.-K. Yang, and H.-G. Kim, "Social semantic cloud of tag: Semantic model for social tagging," in Agent and Multi-Agent Systems: Technologies and Applications, ser. Lecture Notes in Computer Science, N. Nguyen, G. Jo, R. Howlett, and L. Jain, Eds. Springer Berlin / Heidelberg, 2008, vol. 4953, pp. 83-92.

[12] R. Newman, D. Ayers, and S. Russell, "Tag ontology," December 2005. [Online]. Available: http://www.holygoat. co.uk/owl/redwood/0.1/tags/

[13] m.c. schraefel, D. Smith, A. Russell, and M. Wilson, "Semantic web meets web 2.0 (and vice versa): The value of the mundane for the semantic web," PsycPrints (United Kingdom), Tech. Rep., 2006. 
[14] J. Broekstra, A. Kampman, and F. van Harmelen, "Sesame: A generic architecture for storing and querying rdf and rdf schema," in International Semantic Web Conference, ser. Lecture Notes in Computer Science, I. Horrocks and J. A. Hendler, Eds., vol. 2342. Springer, June 9-12 2002, pp. 54-68.

[15] B. McBride, "Jena: A semantic web toolkit," IEEE Internet Computing, vol. 6, no. 6, pp. 55-59, 2002.

[16] D. Torres, H. Skaf-Molli, A. Diaz, and P. Molli, "Supporting personal semantic annotations in p2p? semantic wikis," in 20th International Conference on Database and Expert Systems Applications- DEXA 2009, ser. Lecture Notes in Computer Science, vol. 5690. Linz, Austria: Springer, August 2009.

[17] J. Brooke, "SUS: A quick and dirty usability scale," in $U s-$ ability evaluation in industry, P. W. Jordan, B. Weerdmeester, A. Thomas, and I. L. Mclelland, Eds. London: Taylor and Francis, 1996.

[18] A. Bangor, P. T. Kortum, and J. T. Miller, "An Empirical Evaluation of the System Usability Scale," International Journal of Human-Computer Interaction, vol. 24, no. 6, pp. 574-594, 2008.

[19] C. Bizer, A. Jentzsch, and R. Cyganiak. (2010, Oct.) State of the lod cloud. [Online]. Available: http://www4.wiwiss. fu-berlin.de/lodcloud/state/

[20] C. Bizer, J. Lehmann, G. Kobilarov, S. Auer, C. Becker, R. Cyganiak, and S. Hellmann, "Dbpedia - a crystallization point for the web of data," Journal Web Semantics: Science, Services and Agents on the World Wide Web, vol. 7, no. 3, pp. 154-165, 2009.

[21] J. Kahan and M.-R. Koivunen, "Annotea: an open rdf infrastructure for shared web annotations," in Proceedings of the 10th international conference on World Wide Web, ser. WWW '01. New York, NY, USA: ACM, 2001, pp. 623-632.

[22] S. Mukherjee, I. V. Ramakrishnan, and M. Kifer, "Semantic bookmarking for non-visual web access," in Proceedings of the 6th international ACM SIGACCESS conference on Computers and accessibility, ser. Assets '04. New York, NY, USA: ACM, 2004, pp. 185-192.

[23] V. Zacharias and S. Braun, "Soboleo - social bookmarking and lighweight engineering of ontologies," in Proceedings of the Workshop on Social and Collaborative Construction of Structured Knowledge (CKC 2007) at the 16th International World Wide Web Conference (WWW2007), ser. CEUR Workshop Proceedings, N. F. Noy, H. Alani, G. Stumme, P. Mika, Y. Sure, and D. Vrandecic, Eds., vol. 273. CEUR-WS.org, May 82007.

[24] F. Limpens, "Multi-points of view enrichment of folksonomies," PhD, Université Nice - Sophia Antipolis, October 2010. [Online]. Available: http: //tel.archives-ouvertes.fr/tel-00530714/fr/

[25] A. Monnin, F. Limpens, F. Gandon, and D. Laniado, "Speech acts meet tagging: Nicetag ontology," in Proceedings of the 6th International Conference on Semantic Systems, ser. ISEMANTICS '10. New York, NY, USA: ACM, 2010, pp. $31: 1-31: 10$
[26] Benjamin Huynh-Kim Bang, Eric Dané, and Monique Grandbastien, "Merging semantic and participative approaches for organising teachers' documents," in Proceedings of ED-Media 08 ED-MEDIA 08 - World Conference on Educational Multimedia, Hypermedia \& Telecommunications, Vienna France, 07 2008, pp. p. 4959-4966.

[27] K. Siorpaes and M. Hepp, "Ontogame: Weaving the semantic web by online games," in The Semantic Web: Research and Applications, 5th European Semantic Web Conference, ESWC 2008, ser. Lecture Notes in Computer Science, S. Bechhofer, M. Hauswirth, J. Hoffmann, and M. Koubarakis, Eds., vol. 5021. Springer, June 1-5 2008, pp. 751-766.

[28] H. L. Kim, S. Scerri, J. G. Breslin, S. Decker, H. G. Kim, J.-R. Gu, and Y.-G. Dong, "The State of the Art in Tag Ontologies: A Semantic Model for Tagging and Folksonomies," in International Conference on Dublin Core and Metadata Applications, DC-2008-Berlin Proceedings, September 2008, pp. $128-137$.

[29] A. Passant and P. Laublet, "Meaning Of A Tag: A collaborative approach to bridge the gap between tagging and Linked Data," Proceedings of the WWW 2008 Workshop Linked Data on the Web (LDOW2008), Beijing, China, Apr, 2008.

[30] L. Meiyu, D. Junping, J. Yingmin, and S. Zengqi, "Image semantic description and automatic semantic annotation," in Control Automation and Systems (ICCAS), 2010 International Conference on, ser. IEEE Xplore. IEEE Xplore, 2010, pp. 1192-1195. 